\title{
Les pratiques parentales, le désengagement scolaire des amis et le rendement scolaire chez les élèves du secondaire nés à Haïti et fréquentant une école en milieu défavorisé Parental Practices, Peers' Disengagement from School and Achievement among Haitian youth from low socioeconomical backgrounds
}

\author{
Kristel Tardif-Grenier, Isabelle Archambault et Michel Janosz
}

Volume 40, numéro 2, 2011

URI : https://id.erudit.org/iderudit/1061848ar

DOI : https://doi.org/10.7202/1061848ar

Aller au sommaire du numéro

Éditeur(s)

Revue de Psychoéducation

ISSN

1713-1782 (imprimé)

2371-6053 (numérique)

Découvrir la revue

Citer cet article

Tardif-Grenier, K., Archambault, I. \& Janosz, M. (2011). Les pratiques parentales, le désengagement scolaire des amis et le rendement scolaire chez les élèves du secondaire nés à Haïti et fréquentant une école en milieu défavorisé. Revue de psychoéducation, 40(2), 261-282.

https://doi.org/10.7202/1061848ar

\section{Résumé de l'article}

Les adolescents d'origine haïtienne provenant de milieux défavorisés sont particulièrement à risque d'avoir un faible rendement scolaire. Pourtant, peu d'études canadiennes se sont intéressées au vécu scolaire des élèves de cette communauté. La présente étude a évalué l'effet modérateur de quatre pratiques parentales (règles parentales, aide aux devoirs, communication parent-école et soutien scolaire) sur la relation entre le désengagement scolaire des amis et le rendement en français d'adolescents haïtiens. Cette étude longitudinale compte 467 élèves de secondaire I, nés en Haïti et fréquentant des écoles secondaires situées dans des milieux défavorisés de la région de Montréal. Les résultats ont montré que le désengagement scolaire des amis, les règles parentales, le soutien scolaire et la communication parent-école prédisent significativement le rendement scolaire. Toutefois, aucune des quatre pratiques parentales ne modère le lien entre le désengagement scolaire des amis et le rendement de l'élève. Ces résultats mettent en évidence l'importance de considérer les parents et les amis de l'élève pour comprendre les difficultés scolaires des adolescents haïtiens provenant de milieux défavorisés. Il serait souhaitable que les programmes d'intervention et de prévention visant à augmenter les chances de succès scolaire de ces jeunes impliquent leurs parents et leurs amis.
Tous droits réservés ( $\odot$ La Corporation de la Revue Canadienne de Psycho-Éducation, 2011
Ce document est protégé par la loi sur le droit d'auteur. L'utilisation des services d'Érudit (y compris la reproduction) est assujettie à sa politique d'utilisation que vous pouvez consulter en ligne. 


\section{Les pratiques parentales, le désengagement scolaire des amis et le rendement scolaire chez les élèves du secondaire nés à Haïti et fréquentant une école en milieu défavorisé}

\section{Parental Practices, Peers' Disengagement from School and Achievement among Haitian youth from low socioeconomical backgrounds}

\section{K. Tardif-Grenier ${ }^{1}$ I. Archambault ${ }^{1}$ M. Janosz ${ }^{1}$}

1. École de psychoéducation, Université de Montréal.

Correspondance :

Kristel Tardif-Grenier

École de Psychoéducation

Université de Montréal

C.P. succursale Centre-Ville

Montréal, Québec

H3C 3J7

Téléphone 514 343-6111, poste 50174

k.tardif-grenier@umontreal.ca
Résumé

Les adolescents d'origine haïtienne provenant de milieux défavorisés sont particulièrement à risque d'avoir un faible rendement scolaire. Pourtant, peu d'études canadiennes se sont intéressées au vécu scolaire des élèves de cette communauté. La présente étude a évalué l'effet modérateur de quatre pratiques parentales (règles parentales, aide aux devoirs, communication parent-école et soutien scolaire) sur la relation entre le désengagement scolaire des amis et le rendement en français d'adolescents haïtiens. Cette étude longitudinale compte 467 élèves de secondaire I, nés en Haïti et fréquentant des écoles secondaires situées dans des milieux défavorisés de la région de Montréal. Les résultats ont montré que le désengagement scolaire des amis, les règles parentales, le soutien scolaire et la communication parent-école prédisent significativement le rendement scolaire. Toutefois, aucune des quatre pratiques parentales ne modère le lien entre le désengagement scolaire des amis et le rendement de l'élève. Ces résultats mettent en évidence l'importance de considérer les parents et les amis de l'élève pour comprendre les difficultés scolaires des adolescents haïtiens provenant de milieux défavorisés. II serait souhaitable que les programmes d'intervention et de prévention visant à augmenter les chances de succès scolaire de ces jeunes impliquent leurs parents et leurs amis.

Mots-clés : Rendement scolaire, pratiques parentales, désengagement scolaire des amis, adolescents haïtiens, pauvreté.

\section{Abstract}

Haitian adolescents coming from low socioeconomical backgrounds are particularly at risk for school underachievement. Yet, few Canadian studies looked at the academic experience of students from this community. The present study assessed the moderating effect of four school-related parental practices (monitoring, help with homework, communication with school and, school 


\section{2}

support) on the relationship between peers' disengagement from school and Haitian adolescent achievement in literacy. We used a longitudinal sample of 467 seventh graders born in Haiti and coming from economically disadvantaged high schools of the Montreal area. Results showed that peers' academic disengagement, parental monitoring, school support and, communication with school reliably predicted school achievement. However, none of the four parental practices moderated the link between peers' disengagement with school and student achievement. Our findings highlight the importance of considering parental and peer factors in explaining the achievement of economically disadvantaged Haitian adolescents. It is worthy that intervention programs and preventive measures aimed at increasing the educational achievement of these students involve their parents and friends.

\section{Key words: School achievement, Parental practices, Peers' disengagement from school, Haitian adolescents, Poverty.}

Les difficultés scolaires, c'est-à-dire vivre un échec scolaire ou abandonner l'école avant d'avoir obtenu un diplôme d'études secondaires, ont des conséquences psychosociales et économiques importantes. Des études ont montré qu'un élève qui a de faibles résultats à l'école est plus à risque d'abus de substances, de dépression et de délinquance (Cairns, Cairns, \& Neckerman. 1989; Dryfoos, 1996). Les difficultés académiques constituent également une source importante de tensions familiales et de stress pour l'adolescent (Claes \& Comeau, 1996). À long terme, les difficultés scolaires génèrent des coûts sociétaux importants en contribuant à hausser le taux de chômage et de criminalité (Dryfoos, 1996). Les difficultés académiques ne touchent cependant pas tous les élèves de la même façon. Elles sont influencées par plusieurs facteurs individuels, mais également par des variables liées à la famille et aux amis (Ascher, 1988; Berndt, 1999; Spera, 2005). Dans la présente étude, nous aborderons l'effet des pratiques parentales comme modérateur de la relation entre le désengagement scolaire des amis et le rendement scolaire en français chez les élèves d'origine haïtienne provenant de milieux défavorisés. La communauté haïtienne étant très présente au Québec, une meilleure compréhension de ces facteurs pourrait permettre d'orienter les mesures préventives et les interventions à privilégier afin de favoriser la réussite scolaire des jeunes de cette communauté.

\section{Immigration, pauvreté et difficultés scolaires}

Les enfants et les adolescents issus de l'immigration récente, c'est-à-dire qui sont nés à l'étranger ou dont les parents sont nés à l'étranger, sont souvent confrontés à de nombreux défis d'adaptation découlant de la situation d'immigration. En effet, il est fréquent que ces jeunes doivent apprendre une nouvelle langue et entamer leur parcours scolaire en classe d'accueil (Matte \& Saint-Jacques, 2000). De plus, il arrive que les membres de la famille immigrante s'adaptent à des rythmes différents à la culture du pays d'accueil ou qu'ils éprouvent un bouleversement de leur rôle provoqué par l'exposition à des valeurs différentes de celles ayant cours dans leur pays d'origine (Kanouté, 2002) et il est possible que cela représente une source de stress pour l'élève. Pour plusieurs de ces jeunes, les défis de l'immigration se conjuguent à des conditions socioéconomiques précaires qui rendent cette transition d'autant plus difficile (Van Ngo \& Schleifer 
2005). Additionnées, ces difficultés peuvent avoir d'importantes répercussions sur la réussite scolaire des jeunes (Hicks, 2001; Reynolds, 1991) et ce, au-delà de facteurs de risque connus tels que, par exemple, le sexe, le quotient intellectuel et les problèmes de comportement

Les études portant sur la réussite académique des jeunes issus de l'immigration qui vivent en milieux défavorisés sont conduites pour la plupart aux États-Unis. Elles comparent généralement le rendement scolaire d'élèves issus de différents groupes ethniques (Mau, 1997; Sanders, 1998; Stevenson, Chen, \& Uttal, 1990). Ces études allèguent entre autres que les jeunes afro-américains auraient un rendement scolaire inférieur aux autres groupes ethniques et que les jeunes asiatiques obtiendraient des résultats académiques supérieurs aux autres groupes. Malgré leur pertinence, ces travaux utilisent souvent comme base de comparaison des groupes hétérogènes, par exemple, les Caucasiens, les Afro-Américains ou les Asiatiques, ce qui limite passablement les conclusions qu'elles permettent de tirer sur la réalité scolaire des jeunes appartenant à une communauté spécifique. En effet, chaque communauté possède une histoire, un bagage culturel et des valeurs qui lui sont propres et ces spécificités sont trop souvent diluées par des tentatives de généralisation approximatives. Afin de contourner en partie cette limite, la présente étude vise à mieux comprendre la réalité scolaire d'un groupe spécifique d'adolescents, soit les jeunes immigrants de première génération d'origine haïtienne qui fréquentent une école secondaire en milieu défavorisé.

\section{Difficultés scolaires chez les élèves haïtiens issus de milieux défavorisés}

Plusieurs études québécoises se sont intéressées entre autres au vécu scolaire des élèves originaires d'Haïti tous statuts socioéconomiques confondus (CHOIS, 1981; Lafortune \& Kanouté, 2007; McAndrew et al., 2008; Robergeau, 2007; Saint-Fleur, 2007). Notamment, une étude longitudinale de McAndrew et al. (2008) menée sur la base de données officielles du Ministère de l'Éducation, des Loisirs et des Sports s'est intéressée à la réussite scolaire des jeunes des communautés noires au secondaire. L'échantillon incluait 2168 élèves montréalais créolophones et originaires des Antilles. Environ $70 \%$ de ces élèves étaient issus de milieux défavorisés (c'est-à-dire qu'ils se situaient dans les déciles huit, neuf et dix de l'indice de milieu socioéconomique du ministère de l'Éducation, des Loisirs et des Sports et plus de $80 \%$ étaient nés hors du Québec. Les résultats de ce rapport suggèrent que les élèves créolophones et originaires des Antilles ayant participé à cette étude affichent un taux de réussite plus faible aux épreuves ministérielles en français écrit $(67,3 \%)$ que l'ensemble de la population (89,6\%). Enfin, $35,3 \%$ des élèves de l'échantillon provenant des Antilles, dont la langue maternelle est le créole et qui sont nés hors du Québec obtiennent un diplôme d'études secondaires, comparativement à $71,3 \%$ chez les élèves nés au Québec. L'une des principales conclusions de ce rapport est que les prédicteurs les plus importants de l'abandon scolaire sont le fait d'être non francophone, de recevoir du soutien linguistique au secondaire, d'être né hors Québec, d'avoir intégré le système scolaire au secondaire et d'être désignée comme élèves handicapés ou élèves en difficulté d'adaptation ou d'apprentissage (EHDAA). De plus, les auteurs proposent un portrait type de l'élève à risque d'éprouver des difficultés scolaires : un élève créolophone ou anglophone d'origine antillaise qui est né hors du Québec, 


\section{4}

qui arrive en début de scolarité secondaire et qui fréquente une école montréalaise située en milieu défavorisé (McAndrew et al., 2008). Par ailleurs, au-delà des caractéristiques sociodémographiques qui pourraient expliquer les difficultés scolaires de ces élèves, d'autres facteurs tels que le désengagement scolaire des amis (Berndt, 1999; Epstein \& Karweit, 1983) et les pratiques parentales (Ascher, 1988; Reynolds, 1991; Spera, 2005) pourraient avoir un impact considérable sur le rendement scolaire des adolescents d'origine haïtienne.

\section{Les difficultés scolaires et le désengagement des amis}

L'adolescence est une période de transition importante au cours de laquelle les amis occupent une place grandissante (Berndt \& Keefe, 1995; Cauce, 1986). En raison d'un besoin croissant d'autonomie, les adolescents sont moins réceptifs à l'ingérence de leurs parents dans leur vie personnelle et scolaire et se tournent davantage vers les amis comme source de soutien et de réconfort (Spera, 2005). L'influence exercée par les amis est souvent positive et peut favoriser l'adaptation sociale et scolaire de l'adolescent (Berndt, 1992). Par contre, pour certains adolescents, les amis peuvent avoir une influence négative, notamment au niveau du rendement et du comportement à l'école (Coleman, 1961; Reynolds, 1991). Parmi les principaux éléments par lesquels l'influence des amis opère, les caractéristiques des amis sont importantes à considérer (Berndt, 1992). En effet, le fait d'avoir des amis désengagés vis-à-vis de l'école, c'est-à-dire qui accordent peu de valeur à l'école ou qui ont abandonné l'école constitue un facteur de risque d'échec et d'abandon scolaire chez les adolescents (Altermatt \& Pomerantz, 2005; Dionne-Taylor, 1999; Kindermann, 2007; Mounts \& Steinberg, 1995). Même si aucune pression négative explicite n'est exercée par les amis, l'élève peut être négativement influencé par des amis qui sont peu intéressés par l'école et qui obtiennent de faibles résultats scolaires (Berndt, 1999). Toutefois, les élèves qui ont un rendement scolaire élevé choisissent le plus souvent des amis qui ont de bonnes notes et un bon comportement en classe (Berndt \& Keefe, 1995; Cairns et al., 1989). De la même manière, les élèves qui ont un rendement scolaire faible ont tendance à choisir des amis qui ont peu d'intérêt à obtenir de bonnes notes et qui ont un comportement perturbateur en classe. Dans les deux cas, les amis vont renforcer des tendances existantes, c'est-à-dire que les élèves ayant un rendement scolaire élevé seront encouragés à s'améliorer et les élèves ayant un rendement scolaire faible seront peu incités à adopter des comportements qui favoriseraient leur réussite scolaire (Berndt, 1999; Kindermann, McCollam, \& Gibson, 1996).

À l'heure actuelle, il existe peu de connaissances sur l'influence exercée par les amis chez les jeunes d'origine haïtienne. Par contre, la théorie de l'acting white développée aux États-Unis par Fordham et Ogbu (1986) pourrait permettre une meilleure compréhension du phénomène. Selon cette théorie, les adolescents noirs sont plus susceptibles d'avoir des amis désengagés au plan scolaire que les élèves caucasiens. En effet, comme il existe moins de modèles noirs de réussite scolaire et que des inégalités sociales assujettissent les Afro-Américains (Fordham \& Ogbu, 1986; Hicks, 2001), ces jeunes savent qu'il leur sera plus difficile d'accéder à des emplois prestigieux même s'ils détiennent la formation requise pour le poste convoité (Chiswick, 2008; Dionne-Taylor, 1999). En réponse à ces inégalités, les jeunes noirs ont développé un sentiment de cohésion exacerbée (fictive kinship) 
avec d'autres jeunes noirs (Fordham \& Ogbu, 1986). Cette cohésion exacerbée se traduit par le regroupement de jeunes noirs et par le rejet des valeurs qu'ils jugent appartenir aux blancs. L'école étant le plus souvent gérée et organisée par des blancs, la réussite scolaire fait partie des valeurs qui sont ainsi rejetées (Fordham \& Ogbu, 1986; Hicks, 2001).

En outre, des études menées aux États-Unis rapportent que pour certains adolescents afro-américains, obtenir de bons résultats scolaires est considéré comme socialement indésirable puisque cela représente une adhésion aux valeurs de la culture caucasienne (Fordham \& Ogbu, 1986; Hicks, 2001). Selon ces études, les élèves afro-américains qui manifestent ouvertement leur désir de réussir à l'école risquent d'être rejetés par leurs pairs afro-américains (DionneTaylor, 1999; Ford, 1993). De plus, il arrive que des élèves afro-américains qui réussissent à l'école se regroupent avec des jeunes d'une autre origine ethnique ou diminuent volontairement leur rendement scolaire (Dionne-Taylor, 1999; Ford, 1993). Par ailleurs, la théorie proposée par Fordham et Ogbu (1986) n'a jamais été formellement testée et la présente étude n'a pas pour objectif de la valider. De plus, le niveau d'affiliation avec des amis désengagés n'a pas été documenté spécifiquement chez les élèves québécois d'origine haïtienne. II est donc possible que le phénomène de cohésion exacerbée observé aux États-Unis ne soit pas présent au Québec. En effet, au Québec, la discrimination raciale existe mais est sans doute moins manifeste qu'aux États-Unis (Robergeau, 2007).

\section{Les difficultés scolaires et les pratiques parentales}

Les parents sont des acteurs cruciaux lorsqu'il est question du rendement scolaire de leur enfant (Ascher, 1988; Deslandes, 2004; Spera, 2005). Leur influence est à considérer dans le cas des élèves issus de l'immigration car bon nombre de parents immigrants perçoivent l'école comme un outil d'ascension sociale et d'intégration à la société d'accueil et par conséquent, y accordent une grande importance (Kanouté, Vatz-Laaroussi, Rachédi, \& Tchimou Doffouchi, 2008; Vatz-Laaroussi, Kanouté, \& Rachédi, 2008). Selon Mau (1997), il existe quatre catégories de pratiques parentales qui influencent le rendement de l'enfant : les règles parentales; le soutien scolaire des parents; l'aide aux devoirs; et la communication école-parents. De plus, la participation parentale à la maison est tout aussi importante que la participation des parents à l'école pour favoriser la réussite scolaire de l'enfant (Deslandes, 2004). Plutôt que d'autres modèles qui rendent compte des caractéristiques parentales (Baumrind, 1966; Epstein, 1996), nous avons choisi le modèle de Mau (1997) parce qu'il a été validé auprès d'un échantillon d'adolescents issus de l'immigration récente. Par ailleurs, étant donné qu'il existe très peu d'études sur les pratiques parentales ayant cours dans les familles haïtiennes, nous documenterons ces quatre catégories de pratiques à partir de résultats de recherches menées auprès de familles afro-américaines. Soulignons toutefois qu'à bien des égards, ces pratiques sont semblables à celles des parents de la population en général.

Les règles parentales. Les règles parentales se définissent par la supervision des activités extrascolaires, comme par exemple la surveillance du temps passé avec les amis ou à regarder la télévision. Une étude de Baumrind 
(1972) incluant 16 enfants afro-américains et leur famille a montré que le style parental autoritaire, c'est-à-dire qui est caractérisé par un contrôle parental élevé et beaucoup de règles familiales, est celui qui est le plus souvent adopté par les parents dont les enfants ont un rendement scolaire élevé. Cela s'expliquerait par le fait que beaucoup de jeunes afro-américains sont susceptibles de vivre dans des quartiers défavorisés où il y des taux élevés de délinquance et de fréquenter des pairs ayant des conduites déviantes (Dionne-Taylor, 1999; Fletcher, 1994). Or, en contrôlant les activités de leur adolescent, les parents afro-américains le mettent en quelque sorte à l'abri de la fréquentation d'amis déviants ou désengagés au niveau scolaire et peuvent l'orienter vers des buts liés à la réussite scolaire (Baumrind, 1972; Fletcher, 1994).

Le soutien scolaire des parents. Le soutien scolaire parental fait référence au soutien que le parent fournit à son adolescent en s'intéressant à ses résultats et à ses activités scolaires (Mau, 1997). Les parents afro-américains qui encouragent leur enfant à fournir des efforts à l'école et qui savent où il en est dans ses apprentissages favorisent la réussite scolaire de celui-ci (Archer \& Francis, 2007; Clark, 1983; Hicks, 2001). De plus, les parents afro-américains qui ont des discussions avec leur enfant sur ce qui a été appris en classe et au sujet de ses émotions en lien l'école augmentent ses chances de réussir à l'école. Enfin, une étude menée auprès de 827 élèves afro-américains du primaire a mis en évidence que les élèves qui réussissent le mieux ont des parents qui tentent de les aider s'ils ont des difficultés avec certains apprentissages (Sanders, 1998).

L'aide aux devoirs. L'aide aux devoirs implique que le parent supervise et aide l'adolescent lorsqu'il fait ses travaux scolaires à la maison. Une recension des écrits sur l'aide aux devoirs dans les familles immigrantes a montré que la supervision parentale des devoirs peut se faire de diverses manières (Hoover-Dempsey, Battiato, Walker, Reed, DeJong, \& Jones, 2001), notamment en structurant le temps et l'espace au sein desquels s'effectuent les devoirs et en profitant de la période des devoirs pour enseigner des stratégies d'apprentissage à l'enfant. Les parents afro-américains qui encadrent la période des devoirs augmentent les chances de réussite scolaire de leur enfant et ceux-ci obtiennent de meilleurs résultats scolaires que les élèves dont les parents ne fournissent pas un tel encadrement en ce qui a trait aux devoirs (Clark, 1993; Hoover-Dempsey et al., 2001). Par contre, on observe que les parents afro-américains dont les enfants échouent à l'école supervisent rarement les devoirs de leurs enfants et se contentent de leur rappeler à l'occasion qu'ils doivent faire leurs devoirs (Clark, 1993). Cependant, l'effet de l'aide aux devoirs sur le rendement de l'élève n'est pas clairement établi et certains auteurs rapportent une absence d'effet de cette pratique sur le rendement scolaire (Balli, Wedman, \& Demo, 1997; McDermott, Goldman, \& Varenne, 1984).

La communication parent-école. La communication parent-école fait référence au contact du parent avec l'école et à sa présence aux rencontres scolaires de même qu'à son implication dans la prise de décisions touchant l'école. II s'agit de la pratique parentale la mieux documentée en ce qui concerne les familles immigrantes en général, et plus particulièrement les familles haïtiennes. Bien que certaines études attribuent les difficultés des parents à entrer en communication avec l'école à la défavorisation (Kurdek \& Fine, 1993; Violette, 1991), d'autres 
travaux ont identifié des facteurs propres aux familles immigrantes. Notamment, Ascher (1988) et Holh (1996) rapportent que les liens qui unissent écoles et familles issues de minorités ethniques sont parfois fragilisés par des attitudes de suspicion et d'incompréhension de part et d'autre. En effet, certaines familles immigrantes, surtout celles qui ont un statut de réfugié, ont peur de s'exprimer devant les intervenants scolaires, les percevant comme des représentants du gouvernement et craignant des représailles ou le renvoi dans leur pays (Hohl, 1996). D'autre part, la faible présence des parents immigrants à l'école est souvent interprétée par les enseignants comme un manque d'intérêt pour l'éducation de leurs enfants. Or, une étude longitudinale menée auprès de 1200 adolescents afro-américains a mis en évidence que les parents qui participent aux activités scolaires véhiculent à leurs adolescents le message que la réussite scolaire est importante pour eux (Fletcher, 1994). Ces adolescents seront alors plus susceptibles de se concentrer sur des objectifs de réussite scolaire et de s'engager dans des activités qui ont pour but la réussite scolaire, comme par exemple la participation à des séances de tutorat à l'école. Par ailleurs, beaucoup de parents d'origine haïtienne ne participent pas à la vie scolaire de leur enfant, souvent par ignorance, par manque de disponibilité ou par peur d'être en contact avec ce qui représente l'autorité dans leur pays d'origine (CHOIS, 1981; Icart, 2010). Également, en Haïti, les aspects touchant le rapport famille-école ne font pas partie des politiques d'éducation et la participation scolaire des parents est surtout financière (Saint-Fleur, 2007). Enfin, une étude menée auprès de parents haïtiens a mis en évidence que le signalement régulier et précoce des difficultés de leur enfant a pour effet de les rassurer quant aux intentions du milieu scolaire et d'exprimer la volonté de l'école de collaborer avec les parents (Icart, 2010). En somme, on constate qu'il existe peu de connaissances sur les pratiques parentales des parents de la communauté haïtienne du Québec. De plus, la plupart des études portant sur les pratiques parentales se sont intéressées aux enfants d'âge scolaire (Ascher, 1988) et peu d'entre elles ont traité des adolescents (Fletcher, 1994; Mau, 1997; Spera, 2005).

\section{Désengagement des amis et difficultés scolaires : effet modérateur des pratiques parentales}

Quelques auteurs affirment qu'étant donné la place grandissante occupée par les amis à l'adolescence, l'influence de la famille décline durant cette phase du développement (Cauce, 1986; Coleman, 1961). Par contre, cette idée demeure peu appuyée au plan empirique. En fait, même si elle peut diminuer, l'influence parentale demeure présente tout au long de l'adolescence (Spera, 2005). Ainsi, il est envisageable que les pratiques parentales puissent moduler l'influence négative des amis sur le rendement scolaire de l'élève. À cet égard, bien que la fréquentation d'amis désengagés au plan scolaire constitue un facteur de risque de l'échec scolaire, certaines pratiques parentales pourraient agir à titre de facteur de protection. Nous pensons que cela sera également vrai pour les élèves nés en Haïti, mais il n'existe toutefois pas de littérature à ce sujet. Par exemple, Mounts et Steinberg (1995) ont montré que le fait d'avoir des parents appliquant des règles claires et démontrant de l'affection peut agir indirectement sur le rendement scolaire en protégeant l'adolescent de l'influence négative de ses amis. En effet, les adolescents sujets à des règles parentales constantes ont moins de possibilités de fréquenter des amis déviants ou désengagés vis-à-vis de l'école puisque leurs parents contrôlent 


\section{8}

et s'informent du temps qu'ils passent avec leurs amis et des activités auxquelles ils se livrent lorsqu'ils sont ensemble. Les parents peuvent donc être favorables ou non aux fréquentations de leur adolescent et choisir de stimuler ou limiter ses contacts avec ses amis (Epstein \& Karweitt, 1983). Par ailleurs, le parent qui aide son adolescent dans ses devoirs encourage ce dernier à se centrer sur une activité favorisant la réussite scolaire plutôt qu'à investir du temps dans des activités moins constructives, telles que flâner avec ses amis (Clark, 1983). Également, le parent qui soutient son adolescent au plan scolaire transmet à ce dernier ses valeurs quant à l'importance de l'instruction et à l'intérêt de fournir des efforts pour parvenir à la réussite (Fletcher, 1994). En étant exposé à de telles valeurs, l'adolescent en vient à les intégrer et devient moins perméable aux pressions exercées par les amis désengagés vis-à-vis de l'école. Enfin, les parents qui communiquent régulièrement avec l'école seront rapidement au fait d'une baisse dans le rendement scolaire ou de la motivation scolaire de leur adolescent (Reynolds, 1991). Advenant que cela soit occasionné par la fréquentation d'amis désengagés, ces parents pourraient être en mesure d'intervenir promptement pour contrecarrer cette influence.

\section{Objectifs et hypothèses}

Par la présente étude, nous souhaitons améliorer les connaissances en ce qui a trait aux facteurs associés au rendement scolaire des élèves nés en Haïti vivant en milieu défavorisé à Montréal. Dans le souci de préciser des cibles d'interventions pertinentes à cette problématique, nous souhaitons mettre en évidence les pratiques parentales qui permettent concrètement d'améliorer les chances de succès scolaire de ces élèves, surtout de ceux qui seraient plus enclins à fréquenter des amis désengagés vis-à-vis de l'école.

Par le biais de cette étude, nous essayons d'évaluer sur une période d'un an l'effet modérateur des pratiques parentales en lien avec l'école sur la relation entre le désengagement scolaire des amis et le rendement scolaire en français chez les élèves du secondaire nés en Haïti et fréquentant une école située en milieu défavorisé. Premièrement, il est attendu que les élèves dont les amis sont désengagés vis-à-vis de l'école rapporteront un rendement scolaire plus faible en français après un an. Deuxièmement, nous anticipons que plus l'adolescent rapporteront que ses parents l'aident dans ses devoirs, lui imposent des règles, le soutiennent au plan scolaire et communiquent avec l'école, plus son rendement en français augmentera après un an. Enfin, troisièmement, nous prévoyons que les pratiques parentales relatives à l'école agiront comme facteur de protection et atténueront le lien entre le désengagement scolaire des amis et les difficultés scolaires en français.

\section{Méthode}

\section{Échantillon}

Les données utilisées dans le cadre de cette étude proviennent de l'évaluation de la "Stratégie d'Intervention Agir Autrement " (SIAA) (Janosz, Archambault, \& Chouinard, 2005). Au printemps 2003, 24738 élèves du secondaire provenant de 69 écoles en milieu défavorisé ont participé à cette évaluation. Dans 
la présente étude, l'échantillon est constitué de 467 élèves de secondaire I nés en Haïti. L'âge moyen des participants est de 13,74 ans $(\sigma=1,05)$ et $49,9 \%$ sont de sexe féminin $(n=233)$. Les participants sont répartis dans 25 écoles dont plus de $80 \%$ sont situées sur l'île de Montréal. Trois différentes cohortes d'élèves de secondaire I ont été incluses dans l'échantillon. Les élèves de la première cohorte, deuxième et troisième cohorte étaient en secondaire I respectivement en 2003 ( $n=$ 221), $2004(n=128)$ et $2005(n=118)$.

\section{Procédure}

Les données ont été recueillies à partir de questionnaires autorévélés administrés en deux temps, soit en secondaire I $\left(t_{1}\right)$ et en secondaire II $\left(t_{2}\right)$. Le rendement en français, les pratiques parentales relatives à l'école et le désengagement scolaire des amis ont été mesurés en secondaire I. Le rendement en français chez ces mêmes élèves a été mesuré un an plus tard, lorsqu'ils étaient en secondaire II. Les questionnaires ont été administrés en classe par les enseignants assistés d'auxiliaires de recherche ayant reçu une formation préalable. La durée de passation se situait entre 60 et 75 minutes. Un consentement parental, sollicité par écrit ou par téléphone, devait être obtenu pour tous les élèves de moins de dix-huit ans. Le taux moyen de consentement pour l'ensemble de la collecte de données visant à évaluer la SIAA était de $76 \%$.

\section{Mesures}

Rendement scolaire en français. Cette variable a été mesurée en secondaire I $\left(t_{1}\right)$ et II $\left(t_{2}\right)$ à l'aide d'un item auto-rapporté par l'élève : " au cours de cette année scolaire, quelles sont tes notes moyennes en français (au meilleur de ta connaissance)? " Le choix de réponses se déploie en 14 intervalles, allant de $0 \%$ à $100 \%$ (ex. de $71 \%$ à $75 \%$ ). Une méta analyse de Kuncel, Crede et Thomas (2005) a mis en évidence que les notes auto-rapportées représentent bien les notes réelles d'un élève, surtout si celui-ci a un rendement scolaire élevé.

Désengagement scolaire des amis. Le désengagement scolaire des amis a été mesuré en secondaire I $\left(\mathrm{t}_{1}\right)$ à l'aide d'une échelle tirée du questionnaire sur l'Intégration sociale et personnelle des élèves (Janosz et al., 2005). Cette échelle comporte trois items (" mes meilleur(e)s ami(e)s parlent souvent de lâcher l'école »; « plusieurs de mes meilleur(e)s ami(e)s ont doublé une année » et « mes meilleur(e)s ami(e)s ne vont plus à l'école ») auxquels l'élève répond à l'aide d'une échelle de type Likert en quatre points : faux (1), plutôt faux (2), plutôt vrai (3) ou vrai (4) ( $\alpha=$ $0,60)$.

Perception des pratiques parentales en lien avec l'école. La perception des pratiques parentales a été mesurée à l'aide de quatre échelles. L'échelle "règles parentales » comprend huit items portant sur les règles ayant cours à la maison (par exemple, « $y$-a-t-il un règlement chez toi portant sur ... l'heure où tu dois rentrer le soir, ... les ami(e)s avec lesquels tu peux sortir », etc.). L'élève répond à ces items par oui (1) ou non ( 0$)(\alpha=0,68)$. L'échelle « soutien scolaire des parents » comprend sept items concernant le soutien scolaire parental (par exemple, « combien de fois, depuis septembre, un de tes parents a-t-il réalisé les actions suivantes : ...me félicite 


\section{0}

pour mes réalisations, ...lorsque j'ai une mauvaise note à l'école m'encourage à faire encore plus d'efforts ", etc.). L'élève répond à l'aide d'une échelle de type Likert en quatre points : jamais $(0)$ à très souvent $(3)(\alpha=0,84)$. L'échelle « aide aux devoirs ॥ comprend quatre items rattachés à l'aide aux devoirs (par exemple, « combien de fois, depuis septembre, un de tes parents a-t-il réalisé les actions suivantes : ...m'aide quand je ne comprends pas quelque chose dans mes travaux scolaires, ...m'aide à faire mes devoirs quand je le demande », etc.). L'élève répond à l'aide d'une échelle de type Likert en quatre points : jamais (0) à très souvent (3) $(\alpha=0,73)$. L'échelle « communication parent-école » est composée de six items concernant la communication des parents avec l'école (par exemple, « combien de fois, depuis septembre, un de tes parents a-t-il réalisé les actions suivantes : ...parle avec tous mes enseignant(e)s, ... rencontre un(e) de mes enseignant(e)s » etc.). L'élève répond à ces questions à l'aide d'une échelle de type Likert en quatre points : jamais $(0)$ à très souvent $(3)(\alpha=0,77)$.

\section{Variables de contrôle}

Statut socioéconomique (SSE). Le SSE a été mesuré à l'aide de l'Indice de richesse à la maison (WEALTH) (Janosz et al., 2005). Cet indice consiste en dixsept énoncés répartis en deux dimensions : la présence de biens dans la maison et le nombre de biens. Premièrement, «dans la maison où tu vis le plus souvent, retrouve-t-on ... un lave-vaisselle, ... une chambre à toi seul, ... une connexion internet? ". L'élève répond à ces questions par oui (1) ou non (0). Deuxièmement, « dans la maison où tu vis le plus souvent, combien y-a-t-il de ... téléviseurs, ... véhicules motorisés, ... salles de bains, ...d'ordinateurs ? ». L'élève répond à cette question à l'aide d'une échelle de type Likert en quatre points : aucun(e) (0) à trois et plus (3). Les deux dimensions ont été combinées pour créer une échelle globale $(\alpha=0,63)$.

Quotient intellectuel (QI). Le QI a été mesuré à l'aide des 59 matrices de Raven (Raven, Raven, \& Court, 2000). II s'agit d'une mesure largement reconnue pour estimer le QI non-verbal. L'élève répond à chaque problème en choisissant une réponse parmi huit possibles dont seulement une est exacte. À l'aide de ces mesures, nous avons obtenu une estimation du QI de chaque participant. Toutefois, la mesure utilisée est un score brut.

Sexe. Le sexe a été évalué à partir d'une question auto-révélée : masculin (1) ou féminin (2).

Inadaptation scolaire. L'inadaptation scolaire a été mesurée à l'aide d'une échelle tirée du questionnaire sur l'Intégration personnelle et sociale des élèves (Janosz et al., 2005). Cette échelle comprend cinq items (par exemple, «au cours des douze derniers mois : .... as-tu manqué l'école sans excuse valable, ....as-tu dérangé ta classe par exprès, ... as-tu répondu à un enseignant en n'étant pas poli »). L'élève répond à ces questions à l'aide d'une échelle en quatre points : jamais $(0)$ à très souvent $(3)(\alpha=0,71)$. 


\section{Stratégie analytique}

Les données ont été analysées en deux étapes. D'abord à l'aide de statistiques descriptives et de corrélations, puis à partir d'une régression linéaire multiple hiérarchique. Les variables ont été introduites en trois modèles : (1) variables de contrôle, (2) désengagement scolaire des amis et (3) pratiques parentales. Les effets modérateurs ont été testés séparément en suivant les principes proposés par Aiken et West (1991). Selon ces principes, la variable modératrice est créée par la multiplication de la variable indépendante et de la variable modératrice (par exemple, le désengagement scolaire des amis $\mathrm{X}$ la perception des règles parentales).

\section{Valeurs manquantes}

Nous avons effectué de l'imputation multiple à l'aide du logiciel NORM afin de remplacer les valeurs manquantes (13 à $59 \%$ selon la variable) (Graham, 2009; Schafer, 1999). Dans le but de comparer les valeurs imputées à celles qui n'ont pas été imputées, les moyennes et les écarts types de chacune des variables avant et après l'imputation ont été examinées. Aucune différence entre les valeurs obtenues pour les deux échantillons n'a été observée. Des analyses plus approfondies indiquent cependant que les élèves ayant des valeurs complètes sont plus souvent les filles $(t(465)=2,04, p<0,05)$ et obtiennent un rendement scolaire en français plus élevé en secondaire I $(t(264)=2,74, p<0,01)$. Dans les deux cas, l'ampleur d'effet est cependant très faible $(d=0,016)$ (Cohen, 1988).

\section{Résultats}

\section{Corrélations simples}

Les relations qui existent entre les variables à l'étude ont d'abord été évaluées à partir de corrélations simples. L'examen de la matrice de corrélation présentée au tableau 1 indique que, de façon générale, les variables sont faiblement à moyennement corrélées entre elles (Cohen, 1988). Contrairement à ce que l'on pourrait présumer, il n'y a pas de corrélation entre le QI, le SSE et le rendement scolaire mesuré au temps 2. II existe toutefois une corrélation entre le SSE et le QI et le rendement scolaire antérieur mesuré au temps 1 .

\section{Régression linéaire multiple}

Effets principaux. Les relations qui existent entre les variables ont été évaluées à l'aide de régression linéaire multiple hiérarchique (Tableau 2). Le premier modèle $(F(5,46)=27,17, p<0,001)$ qui inclut uniquement les variables de contrôle est significatif et rend compte de $22,80 \%$ de la variance du rendement en français en secondaire II. Plus spécifiquement, ce modèle indique que les filles et les élèves qui ont un rendement élevé en français en secondaire I ont un meilleur rendement en français en secondaire II. Curieusement, ce modèle indique que les élèves ayant un faible QI ont un rendement en français plus élevé que les élèves ayant un QI élevé. Cela semble attribuable à un effet de suppression négatif, c'est-à-dire à l'effet d'une autre variable indépendante qui, une fois incluse dans le modèle, supprime la portion de variance qui est sans rapport avec la prédiction de la 
Tableau 1. Matrice des corrélations

\begin{tabular}{|c|c|c|c|c|c|c|c|c|c|c|c|}
\hline & 1 & 2 & 3 & 4 & 5 & 6 & 7 & 8 & 9 & 10 & 11 \\
\hline 1. Rendement $t_{1}$ & 1 & $0,41^{* * *}$ & $-0,21^{* *}$ & 0,02 & $0,10^{*}$ & 0,07 & $-0,07$ & $0,14^{* *}$ & $0,16^{* * *}$ & $0,17^{* * *}$ & $-0,10^{*}$ \\
\hline 2. Rendement $t_{2}$ & & 1 & $-0,33^{* * *}$ & $0,21^{* * *}$ & $0,17^{* * *}$ & $0,14^{* *}$ & $-0,10^{*}$ & 0,05 & $-0,02$ & $0,28^{* * *}$ & $-0,12^{* *}$ \\
\hline 3. Désengagement & & & 1 & $-0,07$ & $-0,13^{* *}$ & $-0,01$ & $0,13^{* *}$ & 0,01 & $-0,08$ & $-0,11^{*}$ & $0,17^{* *}$ \\
\hline 4. Règles parentales & & & & 1 & $0,15^{\star *}$ & $0,20^{* * *}$ & 0,04 & $-0,07$ & $0,10^{*}$ & $-0,07$ & $-0,05$ \\
\hline 5. Aide aux devoirs & & & & & 1 & $0,75^{\star \star *}$ & $0,33^{* * *}$ & $-0,11^{*}$ & 0,09 & $0,22^{* \star *}$ & $-0,21^{* *}$ \\
\hline 6. Soutien scolaire & & & & & & 1 & $0,46^{* * *}$ & $-0,01$ & 0,03 & $0,12^{*}$ & $-0,15^{* *}$ \\
\hline 7. Communication & & & & & & & 1 & $-0,04$ & $-0,21^{* * *}$ & 0,03 & 0,05 \\
\hline 8. SSE & & & & & & & & 1 & $0,16^{\star * *}$ & $-0,02$ & $-0,16^{* *}$ \\
\hline 9. QI & & & & & & & & & 1 & $0,10^{*}$ & $-0,14^{* *}$ \\
\hline 10. Sexe & & & & & & & & & & 1 & $0,01^{\text {** }}$ \\
\hline 11. Inadaptation & & & & & & & & & & & 1 \\
\hline
\end{tabular}

${ }^{*} p<0,05,{ }^{* *} p<0,01,{ }^{* * *} p<0,001$ 
Tableau 2. Résultats de la régression linéaire multiple hiérarchique

\begin{tabular}{lccc}
\hline & Modèle 1 & Modèle 2 & Modèle 3 \\
& $\beta$ & $\beta$ & $\beta$ \\
\hline Intercepte & 45,37 & 53,89 & 50,42 \\
Inadaptation scolaire & $-0,06$ & $-0,03$ & $-0,01$ \\
SES & 0,02 & 0,03 & 0,06 \\
QI & $-0,12^{* *}$ & $-0,13^{* *}$ & $-0,18^{* * *}$ \\
Sexe & $0,22^{* * *}$ & $0,21^{* * *}$ & $0,21^{* * *}$ \\
Rendement $t_{1}$ & $0,38^{* * *}$ & $0,33^{* * *}$ & $0,32^{* * *}$ \\
Désengagement & & $-0,25^{* * *}$ & $-0,22^{* * *}$ \\
Règles parentales & & & $0,20^{* * *}$ \\
Soutien scolaire & & & 0,12 \\
Aide aux devoirs & & & 0,03 \\
Communication & & & $-0,17^{* * *}$ \\
$R 2$ & & 0,28 & 0,35 \\
$\Delta R 2$ & 0,23 & 0,06 & 0,07 \\
\hline${ }^{*} \mathrm{p}<0,05,{ }^{* *} \mathrm{p}<0,01,{ }^{* * *} \mathrm{p}<0,001$ & &
\end{tabular}

variable dépendante (Tabachnick \& Fidell, 2007). Dans le cas présent, la corrélation que le QI entretient avec d'autres variables, notamment le rendement en secondaire I, augmenterait son poids de régression. Enfin, ce modèle indique que l'inadaptation scolaire et le SSE ne sont pas des prédicteurs significatifs du rendement en français.

Le second modèle ajoute le désengagement scolaire des amis. Ce modèle $(F(6,46)=30,29, p<0,001)$ est significatif et permet d'expliquer $28,30 \%$ de la variance du rendement en français en secondaire II. Plus spécifiquement, les résultats indiquent qu'au-delà des variables de contrôle, plus les amis sont désengagés au plan scolaire, moins le rendement de l'élève en français est élevé en secondaire II.

Les échelles mesurant la perception des pratiques parentales ont été ajoutées au troisième modèle. Ce modèle est significatif $(F(10,45)=24,60, p<$ 0,001 ) et permet d'expliquer $35,0 \%$ de la variance du rendement scolaire en français en secondaire II. Les résultats indiquent qu'au-delà des variables de contrôle et du désengagement scolaire des amis, plus l'élève perçoit que ses parents imposent des règles et lui offrent du soutien scolaire, plus il aura un rendement élevé en français 


\section{4}

en secondaire II. Toutefois, plus l'élève perçoit que ses parents communiquent avec l'école, plus il a tendance à avoir un rendement faible en français en secondaire II. Enfin, la perception de l'aide aux devoirs n'apparaît pas comme un prédicteur significatif du rendement en français en secondaire II.

Effets modérateurs. Les effets modérateurs ont été testés séparément en suivant les principes proposés par Aiken et West (1991). Selon ces principes, la variable modératrice est créée par la multiplication de la variable indépendante et du modérateur (par exemple, le désengagement scolaire des amis $\mathrm{X}$ la perception des règles parentales). Les effets modérateurs ont été testés séparément. La régression suggère que toutes les variables d'interaction entre la perception des pratiques parentales et le désengagement scolaire des amis sont non-significatives. Ainsi, aucune des pratiques parentales perçues ne permet de modérer le lien entre le désengagement scolaire des amis et le rendement en français en secondaire II.

\section{Discussion}

L'objectif de la présente étude était d'évaluer l'effet modérateur des pratiques parentales sur la relation entre le désengagement scolaire des amis et le rendement scolaire en français chez des élèves du secondaire nés en Haïti et fréquentant une école de milieu défavorisé. Plus spécifiquement, nous avons examiné si le fait que les parents encouragent et soutiennent leur adolescent dans ses apprentissages, l'accompagnent dans ses devoirs, lui imposent un certain nombre de règles et communiquent sur une base fréquente avec les intervenants de l'école peut le protéger contre l'influence négative d'amis désengagés vis-àvis de l'école. Globalement, nos résultats suggèrent quelques mesures pour la prévention et l'intervention psychoéducative auprès des jeunes de la communauté haïtienne vivant en milieu défavorisé.

Par rapport à la recherche antérieure (Altermatt \& Pomerantz, 2005; Kindermann, 2007, Mounts \& Steinberg, 1995), nos résultats indiquent que plus les amis sont désengagés vis-à-vis de l'école, plus l'élève haïtien est à risque de vivre des difficultés scolaires en français. Plus précisément, cela signifie que les élèves dont les amis ont repris une année scolaire, parlent de quitter l'école ou ont abandonné l'école sont plus à risque d'avoir un faible rendement en français. Ce résultat ne constitue pas une surprise étant donné qu'au cours de l'adolescence, les amis occupent une place importante et sont donc susceptibles d'influencer le vécu scolaire de l'adolescent (Epstein \& Karweitt, 1983). De plus, selon la théorie de l'acting white (Fordham \& Ogbu, 1986), certains jeunes noirs peuvent se regrouper entre eux et rejeter des valeurs qu'ils perçoivent comme appartenant aux blancs comme la réussite scolaire. Selon cette théorie, les jeunes noirs rejettent ces valeurs en réponse aux inégalités raciales auxquelles ils sont assujettis. Bien que la validité de cette théorie reste à être démontrée, si elle s'applique aux jeunes haïtiens de milieux défavorisés nouvellement arrivés au Québec, il est probable que ces derniers aient tendance à s'affilier à des pairs qui dévalorisent l'école et qui sont peu engagés au plan scolaire, ce qui pourrait influencer leur rendement académique. 
D'autre part, nos résultats suggèrent que certaines pratiques adoptées par les parents haïtiens favorisent le rendement scolaire de leur adolescent. Cela vient supporter l'idée que les parents haïtiens demeurent des acteurs importants de la réussite scolaire durant l'adolescence (Mau, 1997; Sanders, 1998). Selon certaines études (Clark, 1983; Mau, 1997), les parents qui encouragent leur adolescent à faire des efforts à l'école, qui ont régulièrement des conversations au sujet de l'école et qui appliquent des règles à la maison contribuent de manière significative à sa réussite scolaire. Notons au passage qu'à bien des égards, ces pratiques sont similaires à celles adoptées par la population en général.

À l'inverse, plus le parent communique sur une base régulière avec les différents intervenants scolaires, plus l'adolescent rapporte un faible rendement. Cela est conforme aux résultats des études sur les relations entre la famille immigrante et l'école (CHOIS, 1981, Clark, 1983, Holh, 1996) de même qu'avec la littérature sur les parents en général (Deslandes \& Lafortune, 2001; Deslandes, Royer, Turcotte, \& Bertrand, 1997). En effet, beaucoup de parents immigrants défavorisés socioéconomiquement sont peu enclins à initier la communication avec l'école de leur enfant. Étant donné que les enseignants communiquent la plupart du temps avec les parents pour rapporter les difficultés de l'élève, des interactions fréquentes entre ces familles et l'école sont plus susceptibles de se produire si l'élève présente des difficultés (Deslandes, 1996). Ainsi, les parents d'élèves dont le rendement en français est plus faible entreront plus souvent en communication avec l'école de leur enfant que les parents dont l'enfant n'éprouve pas de difficulté particulière à l'école. L'augmentation de la fréquence des contacts entre la famille et l'école lorsque l'élève vit des difficultés est une bonne chose puisqu'elle témoigne d'un souci de prise en charge de l'élève en difficulté. Par ailleurs, la tendance à entrer peu fréquemment en communication avec l'école sans y être convoqué est également présente dans la population en général et plus particulièrement chez les familles défavorisées au plan socioéconomique.

Contrairement à ce qui était attendu, nos résultats montrent que l'aide aux devoirs qu'offre le parent haïtien à son enfant ne semble pas contribuer au rendement en français de ce dernier. Ce résultat est appuyé par la recherche et certaines études tirent des conclusions similaires pour la population en général (Balli et al., 1997; McDermott et al., 1984). Selon McDermott et al. (1984). Cette absence d'effet s'expliquerait notamment par l'incompatibilité entre la nature des tâches données en devoir et les besoins individuels de l'élève, mais surtout parce que beaucoup de parents qui tentent d'aider leur enfant ne parviennent pas à le faire de manière efficace. En effet, beaucoup de parents immigrants méconnaissent le fonctionnement du système scolaire québécois et les objectifs visés par l'enseignant (Hohl, 1996). Ils ne savent sans doute pas comment accompagner leur adolescent dans l'atteinte de ces objectifs. Aussi, beaucoup de nouveaux arrivants n'ont pas le français comme langue maternelle et ne sont donc peut-être pas en mesure de lire les consignes et de comprendre les devoirs de leurs enfants (Ministère de I'Immigration et des Communautés culturelles du Québec, 2010). Enfin, comme dans le cas de la communication famille-école, il est envisageable que la défavorisation explique en partie cette absence d'effet de l'aide parentale aux devoirs puisque les parents issus de milieux favorisés sont en mesure d'offrir davantage de soutien aux devoirs (St-Laurent et al., 1994). 
Par ailleurs, les résultats de la présente étude montrent qu'aucune des quatre pratiques parentales en lien avec l'école n'est en mesure d'atténuer l'effet du désengagement des amis sur le rendement en français. Cela est surprenant étant donné que selon certains auteurs, les parents peuvent influencer le niveau d'implication d'un adolescent auprès d'amis déviants en contrôlant le temps qu'il passe avec ses amis et en restreignant ses activités (Dishion, Spracklen, Andrews, \& Patterson, 1996). L'absence d'effet protecteur des pratiques parentales pourrait cependant s'expliquer autrement. On peut envisager que sous l'influence négative de leurs amis, les adolescents parviennent à se soustraire à la supervision parentale et à fréquenter leurs amis en dépit de l'interdiction de leurs parents (Mounts \& Steinberg, 1995). De plus, la déviance des amis peut influencer l'augmentation des conduites antisociales et rendre plus difficile la tâche du parent, ce qui peut engendrer un certain désengagement et du découragement chez le parent (Dishion, Nelson, \& Bullock, 2004). Le parent est alors moins en mesure d'exercer une supervision suffisante pour protéger son adolescent contre l'influence négative de ses amis. Il est également envisageable que l'absence d'effet modérateur découle du fait que les données soient entièrement auto-rapportées par l'élève et que le point de vue des parents et des amis de l'élève ne soient pas pris en compte. Enfin, le nombre élevé de valeurs manquantes fait en sorte que les sujets demeurés dans l'étude sont peut-être moins à risque de présenter des facteurs de risque comme la fréquentation d'amis désengagés au plan scolaire. En l'absence d'un nombre important d'élèves à risque, il est difficile d'observer des effets modérateurs significatifs.

\section{Le rôle du psychoéducateur intervenant auprès des élèves d'origine haïtienne}

De par sa présence dans les milieux scolaires, le psychoéducateur est susceptible d'intervenir auprès d'une clientèle immigrante et il est appelé à favoriser la réussite scolaire et l'intégration des élèves immigrants, dont ceux d'origine haïtienne. Rappelons que le rôle fondamental du psychoéducateur est de mobiliser l'entourage d'un jeune en vue de favoriser l'adaptation de ce dernier (Gendreau, 2001). Étant donné que de nombreux acteurs et facteurs sont impliqués dans le développement des difficultés scolaires (Bronfenbrenner, 1977), l'intervention psychoéducative semble être une avenue à privilégier. En effet, elle est susceptible de rejoindre plusieurs des sphères de développement de l'élève et ainsi de tenir compte de la complexité des facteurs associés à ses difficultés scolaires. À la lumière de nos résultats, nous verrons qu'il est pertinent d'intervenir auprès des amis et des parents de l'élève haïtien afin de favoriser son rendement scolaire.

Intervention auprès des amis. Dans la mesure du possible, le psychoéducateur en milieu scolaire devrait être vigilant quant aux amis fréquentés par l'élève. II serait pertinent de considérer le désengagement scolaire des amis comme un facteur de risque des difficultés scolaires. Le psychoéducateur peut notamment chercher à intervenir auprès des amis en instaurant des mesures favorisant l'engagement scolaire des élèves de toute l'école. À cet égard, selon le modèle de Finn (1989), l'engagement et la participation scolaire des élèves nécessitent un sentiment d'appartenance à l'école de même qu'une appropriation des valeurs de l'école par les élèves. Dans une école où la clientèle haïtienne est très présente, il serait pertinent de mettre en place des activités rassembleuses 
qui interpellent ces jeunes en particulier et qui développent ainsi leur sentiment d'appartenance au milieu, comme par exemple des activités sportives. Par ailleurs, l'élève qui immigre en cours d'adolescence peut éprouver de la difficulté à se faire des amis puisque les autres adolescents ont déjà des réseaux sociaux bien établis. Conséquemment, il est possible que l'élève haïtien nouvellement arrivé se retrouve en situation de rejet et en vienne à fréquenter des pairs marginaux qui sont entre autres désengagés au plan scolaire (Van Ngo \& Schleifer, 2005). II pourrait être pertinent de procéder à un jumelage des nouveaux élèves haïtiens à des pairs ayant un niveau d'engagement scolaire élevé et idéalement d'origine haïtienne permettant ainsi à l'élève haïtien nouvellement arrivé de se construire un réseau social de pairs engagés qui valorisent la réussite scolaire.

Intervention auprès des parents. Concernant l'intervention auprès des parents, il semble pertinent que le psychoéducateur cherche à consolider l'autorité et le soutien scolaire des parents haïtiens. Ainsi, le psychoéducateur pourrait assister les parents haïtiens dont l'adolescent vit des difficultés scolaires dans la mise en place et l'application de règles claires en ce qui a trait aux heures de sorties et à la fréquentation des amis. De plus, le psychoéducateur pourrait outiller et accompagner les parents afin de bonifier leur capacité à soutenir leur adolescent de manière aidante au plan scolaire, notamment par le biais des devoirs. II importe que le psychoéducateur témoigne du respect et de la considération pour les pratiques et les croyances que les parents d'origine haïtienne adoptent afin d'établir un climat de confiance et de non-jugement. Enfin, bien que nos résultats semblent suggérer l'inverse, la communication entre les parents et l'école est un facteur important de succès scolaire chez l'élève et nous pensons que des interventions auprès des parents pourraient viser à améliorer cette communication (Cork, 2005). Dans un premier temps, il serait important de créer des partenariats entre l'école et les organismes communautaires avoisinants. Ainsi, des intervenants pourraient effectuer des visites à domicile qui viseraient à établir un contact initial positif entre la famille et l'école. Au cours de ces visites, l'intervenant pourrait démystifier le fonctionnement de l'école québécoise et les attentes entourant l'implication des parents à l'école puisque ces dernières peuvent différer grandement de celles du pays d'origine de la famille. Enfin, l'intervenant pourrait accompagner les parents lors de leur première visite à l'école afin de les mettre en confiance et d'agir à titre de médiateur entre la famille et les intervenants du milieu scolaire.

\section{Forces et limites de l'étude}

Cette étude comporte plusieurs forces. II s'agit d'abord d'une des rares études quantitatives québécoise à s'intéresser aux difficultés scolaires chez les élèves haïtiens du secondaire. De plus, sa nature longitudinale rend possible la documentation des effets des pratiques parentales et du désengagement des amis dans le temps. Ainsi, elle permet d'examiner si le désengagement des amis et les pratiques parentales peuvent prédire les difficultés scolaires et de tester un effet modérateur. Enfin, contrairement à la plupart des études, celle-ci a pour avantage d'inclure un échantillon relativement homogène au plan ethnique et socioéconomique puisque tous les sujets sont nés en Haïti et vivent en milieux défavorisés. Nous avons ainsi contrôlé deux facteurs de risque importants des difficultés scolaires, soit le fait d'être né à l'extérieur du pays et de provenir d'un milieu défavorisé. 
Malgré son caractère novateur, cette étude comporte également certaines limites. Premièrement, la taille de l'échantillon est relativement petite $(n=467)$. De plus, les écoles où les données ont été recueillies sont toutes situées en milieu défavorisé et principalement localisées sur l'île de Montréal. Ces deux caractéristiques de l'échantillon font en sorte qu'il n'est pas possible de généraliser les résultats obtenus à l'ensemble des jeunes issus de la communauté haïtienne. Deuxièmement, les données ont toutes été rapportées par l'élève ce qui reflète peut-être mal la réalité et il aurait été intéressant d'inclure d'autres informateurs, en particulier les parents. Troisièmement, cette étude considère uniquement le rendement en français. Bien qu'il s'agisse de la matière où on observe le plus de difficulté chez les élèves créolophones originaires des Antilles dans le cadre des épreuves ministérielles (McAndrew et al., 2008), il serait pertinent de considérer d'autres matières comme les mathématiques ou l'anglais. Quatrièmement, comme nous n'avons pas évalué le degré de maîtrise de la langue française par les sujets, il impossible de savoir si un faible rendement en français témoigne d'une difficulté scolaire réelle ou s'il s'agit plutôt d'une conséquence de la non-maîtrise de la langue d'enseignement. Cinquièmement, le temps entre la mesure au $t_{1}$ et la mesure au $t_{2}$ est relativement court et il est probable que davantage de changements auraient pu être observés si les mesures avaient été prises à plusieurs années d'intervalle. Enfin, sixièmement, compte tenu du taux élevé d'attrition, il est probable que l'échantillon soit constitué d'élèves n'éprouvant pas de difficultés particulières parce que les élèves présentant des difficultés scolaires ont possiblement renoncé à participer à l'étude. Conséquemment, les résultats de la présente étude ne reflètent pas le vécu scolaire des élèves nés en Haïti en général, mais d'un sous-groupe avec des caractéristiques particulières.

\section{Implications pour la recherche future}

II serait pertinent d'explorer dans les recherches futures d'autres avenues pour identifier les facteurs permettant de modérer l'effet du désengagement scolaire des amis sur le rendement. Les résultats de la présente étude ont mis en évidence que les pratiques parentales relatives à l'école ne parviennent pas à protéger les adolescents de cette influence. II est donc possible qu'à cet âge, d'autres caractéristiques de nature plus individuelle, par exemple la motivation scolaire ou l'estime de soi, constituent de meilleurs facteurs de protection. II se peut également que d'autres caractéristiques des relations que l'adolescent entretient avec son entourage puissent le protéger du désengagement de ses amis, comme par exemple la qualité de la relation qu'il entretient avec un enseignant ou la qualité de l'attachement avec ses parents. II est donc souhaitable que la recherche future explore ces différentes avenues. Afin d'accroître la généralisation des résultats, les études à venir devraient également considérer d'autres matières que le français et être basées sur des échantillons plus grands et davantage représentatifs de la réalité de la communauté haïtienne au Québec et au Canada. Ainsi, les prochaines études devraient inclure des élèves issus de la grande région de Montréal, mais aussi des banlieues et des régions plus éloignées qui sont susceptibles d'avoir un 
vécu scolaire bien différent (McAndrew et al., 2008). Enfin, des études longitudinales débutant plus tôt, soit dès le début du préscolaire et du primaire seront nécessaires pour observer en amont les facteurs qui permettent de prédire les difficultés scolaires de ces jeunes.

\section{Conclusion}

Nous avons vu que les jeunes Haïtiens vivant en milieu défavorisé sont à risque d'éprouver des difficultés scolaires. Considérant que l'école est un facteur important d'intégration au pays d'accueil, le fait d'y vivre des difficultés rend plus difficile la participation citoyenne et sociale de ces jeunes. Actuellement, il y a peu de programmes d'intervention qui ciblent directement les élèves issus de l'immigration. Or, la société québécoise se targue d'accueillir la diversité et ne cesse d'augmenter les quotas d'immigration sans accroître les ressources qui sont allouées à l'intégration de ces immigrants. Il est essentiel de développer davantage de ressources ainsi que d'acquérir des connaissances sur le vécu des élèves issus de l'immigration afin de favoriser leur intégration scolaire et professionnelle. Ces personnes venues d'ailleurs ont énormément à apporter à la société québécoise et en ne permettant pas leur intégration, nous multiplions les occasions manquées d'enrichir notre société de la richesse de la diversité culturelle.

\section{Références}

Aiken, L.S., \& West, S.G. (1991). Multiple regression: Testing and interpreting interactions. Newbury Park, CA: Sage.

Altermatt, E.R. \& Pomerantz, E.M. (2005). The implications of having highachieving versus low-achieving friends: a longitudinal analysis. Social Development, 14 (1), 61-81.

Archer, L. \& Francis, B. (2007). Understanding minority ethnic achievement: race, gender, class and «success». London: Routeledge.

Ascher, C. (1988). Improving the schoolhome connection for poor and minority urban students. The Urban Review, 20 (2), 109-123.

Balli, S.J., Wedman, J.F., \& Demo, D.H. (1997). Family involvement with middlegrades homework: Effects of differential prompting. Journal of Experimental Education, 66, 31-48.

Baumrind, D. (1966). Effects of authoritative control on child behavior. Child Development, 37, 887-907.

Baumrind, D. (1972). An exploratory study of socialization effects on black children:
Some black-white comparisons. Child Development, 43 (1), 261-267.

Berndt, T.J. (1992). Friendship and friends' influence in adolescence. Current Directions in Psychological Science, 1 (5), 156-159.

Berndt, T.J., \& Keefe, K. (1995). Friends' influence on adolescents' adjustment to school. Child Development, 66 (5), 1312-1329.

Berndt, T.J. (1999). Friends' inflluence on students' adjustment to school. Educational Psychologist, 34 (1), 15-28.

Bronfenbrenner, U. (1977). Toward an experimental ecology of human development. American psychologist, 32 (7), 513-531.

Cairns, R.B., Cairns, B.D., \& Neckerman, H.J. (1989). Early school dropout: Configurations and determinants. Child Development, 60 (6), 1437-1452.

Cauce, A.M. (1986). Social networks and social competences: Exploring the effects of early adolescent friendships. American Journal of Community Psychology, 14 (6), 607-628. 
Centre Haïtien d'Orientation et d'Information Scolaire (CHOIS) (1981). Le jeune Haïtien en milieu scolaire montréalais. Montréal: Conseil Scolaire de l'île de Montréal.

Chiswick, B.R. (1988). Differences in education and earnings across racial and ethnic groups: Tastes, discrimination, and investments in child quality. The Quaterly Journal of Economics, 103 (3), 571-597.

Clark, R. (1983). Family life and school achievement: Why poor black children succeed or fail. Chicago: University of Chicago Press.

Clark, R. (1993). Homework-focused parenting practices that positively affects student achievement. Dans N.F. Chavkin (dir..), Families and schools in a pluralistic society (pp. 57-79). Albany, NY: State University of New York Press.

Claes, M. \& Comeau, J. (1996). L'école et la famille: deux mondes? Lien social et Politiques, 35, 75-85.

Cohen, J.W. (1988). Statistical power analysis for the behavioral sciences $\left(2^{\mathrm{e}}\right.$ éd.). Hillsdale, NJ: Lawrence Erlbaum Associates.

Coleman, J.S. (1961). The adolescent society: The social life of the teenager and its impact on education. New York: Free Press.

Cork, L. (2005). Supporting black pupils and parents: Understanding and improving home-school relations. London: Routeledge.

Deslandes, R. (1996). Collaboration entre l'école et les familles: influence du style parental et de la participation parentale sur la réussite scolaire au secondaire (Thèse de doctorat). Université Laval, Canada.

Deslandes, R. (2004). Collaboration famille-école-communauté : pour une inclusion réussie. Dans $N$. Rousseau \& S. Bélanger (dir.), La pédagogie de l'inclusion scolaire (p. 326-346). Québec, Presses de l'Université du Québec.

Deslandes, R. \& Lafortune, L. (2001). La collaboration école-famille dans l'apprentissage des mathématiques selon la perception des adolescents. Revue des Sciences de l'Éducation, XXVII (3), 649-669.

Deslandes, R., Royer, E., Turcotte, D., \& Bertrand, R. (1997). School achievement at the secondary level: influence of parenting style and parent involvement in schooling. Revue des Sciences de L'éducation de McGill , 32 (3). 191-207.

Dionne-Taylor, E.D. (1999). Do AfricanAmerican adolescents discourage academic excellence? An investigation of the relation between peer support dans academic achievement (Thèse de doctorat). University of Michigan, ÉtatsUnis.

Dishion, T.J., Nelson, S.E., \& Bullock, B.M. (2004). Premature adolescent autonomy: Parent disengagement and deviant peer process in the amplification of problem behaviour, Journal of Adolescence, 27, 515-530.

Dishion, T.J., Spracklen, K.M., Andrews, D.M., \& Patterson, G.R. (1996). Deviancy training in male adolescent friendships. Behavior Therapy, 27 (1), 373-390.

Dryfoos, J.G. (1996). Adolescents at risk: Shaping programs to fit the need. Journal of Negro Education, 65 (1), 5-18.

Epstein, J.L. \& Karweit, N. (1983). Friends in school: Patterns of selection and influence in secondary schools. New York: Academic Press.

Epstein, J.L. (1996). Family-school links: How do they affect educational outcomes? Dans A. Booth et J. Dunn (dir.), Family-school links: How do they affect educational outcomes? Hillsdale, $\mathrm{NJ}$ : Lawrence Erlbaum.

Finn, J.D. (1989). Withdrawing from school. Review of Educational Research, 59 (2), 117-142.

Fletcher, A.C. (1994). Parental and peer influences on the academic achievement of African-American adolescents (Thèse de doctorat). Temple University, ÉtatsUnis. 
Ford, D.Y. (1993). An investigation of the paradox of underachievement among gifted Black students. Roeper Review, 16 (2), 78-84.

Fordham, S. \& Ogbu, J.U. (1986). Black students' school success: Coping with the «burden of acting white». The Urban Review, 18 (3), 176-205.

Gendreau, G. (2001). Jeunes en difficulté et intervention psychoéducative. Montréal: Éditions Science et Culture.

Graham, J.W. (2009). Missing data analysis: Making it work in the real world. Annual Review of Psychology, 60, 549-576.

Hicks, H.S. (2001). A study of factors that influences academic success of black American middle school males (Thèse de doctorat). Virginia Commonwealth University, États-Unis.

Hohl, J. (1996). Qui sont " les parents »? Le rapport de parents immigrants analphabètes à l'école. Lien Social et Politiques, 35, 51-62.

Hoover-Dempsey, K.V., Battiato, A.C., Walker, J.M.T., Reed, R.P., DeJong, J.M. \& Jones, K.P. (2001). Parental involvement in homework. Educational Psychologist, 36(3), 195-209.

Icart, J.C. (2010). Participation parentale et réussite scolaire: les parents des communautés noires dans les écoles secondaires publiques francophones de Montréal. Vivre Ensemble, 17(59), 12-17.

Janosz, M., Archambault, I., \& Chouinard, R. (Mai 2005). Profil descriptif de la réussite des élèves sur le plan de l'instruction, de la socialisation et de la qualification au printemps 2003: tendances nationales pour les écoles SIAA échantillonnées. Équipe de recherche sur les environnements scolaires efficaces (ERESE) et Centre de recherche et d'intervention sur la réussite scolaire (CRIRES), $108 \mathrm{p}$.

Kanouté, F. (2002). Les profils d'acculturation d'élèves issus de l'immigration récente à Montréal. Revue des sciences de l'éducation, 28(1), 171-190.
Kanouté, F., Vatz Laaroussi, M., Rachédi, L., \& Tchimou Doffouchi, M. (2008). Familles et réussite scolaire d'élèves immigrants du secondaire. Revue des Sciences de l'Éducation, 34(2), 265289.

Kindermann, T.A. (2007). Effects of naturally existing peer groups on changes in academic engagement in a cohort of sixth graders, Child Development, 78(4), 1186-1203.

Kindermann, T.A ., McCollam, T.L. \& Gibson, E. (1996). Peer networks and students' classroom engagement during childhood and adolescence. Dans J. Juvonen \& K. R. Wentzel (dir.), Social motivation: Understanding children's school adjustment (pp. 279-312). New York: Cambridge University Press.

Kuncel, N.R., Crede, M., \& Thomas, L. (2005). The validity of self-reported grade point averages, class ranks, and test scores: A meta-analysis and review of the literature. Review of Educational Research, 75(1), 63-82.

Lafortune, G. \& Kanouté, F. (2007). Vécu identitaire d'élèves de première et de deuxième génération d'origine haïtienne. Revue de l'Université de Moncton, 38(2), 33-71.

Matte, J. \& Saint-Jacques, M. (2000). Statistiques et commentaires sur les origines des élèves. 1998-1999 et 19992000. Montréal : Conseil scolaire de l'île de Montréal.

Mau, W.C. (1997). Parental influences on the high school student's academic achievement: A comparison of Asian Immigrants, Asian Americans, and White Americans. Psychology in the Schools, 34(3), 267-277.

McAndrew, M., Ledent, J., \& Ait-Said, R. (2008). La réussite scolaire des jeunes des communautés noires au secondaire. Rapport de recherche. Montréal: Immigration et métropoles.

McDermott, R.P., Goldman, S.V., \& Varenne, H. (1984). When school goes home: Some problems in the organization of homework. Teachers College Record, 85(3), 391-409. 
Mounts, N.S. \& Steinberg, L. (1995). An ecological analysis of peer influence on adolescent grade point average and drug use. Developmental Psychology, 31(6), 915-922.

Raven, J., Raven, J.C., \& Court, J.H. (2000). Standard progressive matrices. England: Oxford Psychologists Press.

Reynolds, A.J. (1991). Early schooling of children at risk. American Educational Research Journal, 28(2), 392-422.

Robergeau, D. (2007). Regards croisés sur la trajectoire scolaire d'élèves d'origine haïtienne en situation de réussite scolaire (Mémoire de maîtrise). Montréal, Université de Montréal.

Saint-Fleur, J.M. (2007). Le contexte prémigratoire et l'implication des parents d'origine haïtienne dans le suivi scolaire de leurs enfants au Québec (Mémoire de maîtrise). Montréal, Université de Montréal.

Sanders, M.G. (1998). The effects of school, family, and community support on the academic achievement of African American adolescents. Urban Education, 33(3), 385-409.

Schafer, J.L. (1999). NORM: Multiple imputation of incomplete multivariate data under a normal model [Logiciel informatique]. University Park: Pennsylvania State University, Département de Statistiques.
Spera, C. (2005). A review of the relationship among parenting practices, parenting styles, and adolescent school achievement. Educational Psychology Review, 17(2), 125-146.

Stevenson, H.W., Chen, C., \& Uttal, D.H. (1990). Beliefs and achievements: A study of Black, White, and Hispanic children. Child Development, 61(2), 508-523.

Tabachnick, B.G. \& Fidell, L.S. (2007). Using multivariate statistics. Boston: Pearson Allyn \& Bacon.

VanNgo, H. \& Schleifer, B. (2005) Regard sur les enfants et les jeunes immigrants. Canadian Issues-Thèmes Canadiens, printemps 2005.

Vatz-Laaroussi, M., Kanouté, F., \& Rachédi, L. (2008). Les divers modèles de collaborations familles immigrantesécoles: de l'implication assignée au partenariat. Revue des sciences de l'éducation, 34(2), 291-311.

Violette, M. (1991). L'école... Facile d'en sortir mais difficile d'y revenir. Enquête auprès des décrocheurs et décrocheuses. Québec : Ministère de l'Éducation, Direction générale de la recherche et du développement.

Worswick, C. (2001). Le rendement scolaire des enfants d'immigrants au Canada. [En ligne]. http://www.statcan.gc.ca/bsolc/ olc-cel/olccel?lang=fra\&catno=11F0019 M20 01178 (Page consultée le 20 juin 2009). 